\title{
Oscillation Criteria for Certain Even Order Neutral Delay Differential Equations
}

\author{
Ruba Al-Hamouri and Ali Zein \\ Department of Applied Mathematics, Palestine Polytechnic University, Hebron, Palestine \\ Correspondence should be addressed to Ali Zein; alizein@ppu.edu
}

Received 20 January 2014; Accepted 1 April 2014; Published 16 April 2014

Academic Editor: Yuriy Rogovchenko

Copyright (C) 2014 R. Al-Hamouri and A. Zein. This is an open access article distributed under the Creative Commons Attribution License, which permits unrestricted use, distribution, and reproduction in any medium, provided the original work is properly cited.

We establish sufficient conditions for the oscillation of solutions of even order neutral type differential equations of the form $\left[r(t)[x(t)+p(t) x(\tau(t))]^{(n-1)}\right]^{\prime}+q(t) f(x(\sigma(t)))=0$.

\section{Introduction}

This paper concerns the oscillatory behavior of solutions of higher order neutral type nonlinear differential equations of the following form:

$$
\begin{aligned}
{\left[r(t)[x(t)+p(t) x(\tau(t))]^{(n-1)}\right]^{\prime}+q(t) f(x(\sigma(t))) } & =0, \\
t & \geq t_{0},
\end{aligned}
$$

where $n \geq 2$ is even and the following conditions are assumed to hold:

(H1) $p, q \in C\left(\left[t_{0}, \infty\right)\right), q(t)>0,0 \leq p(t) \leq p_{0}<1$, where $p_{0}$ is constant;

(H2) $r \in C^{1}\left(\left[t_{0}, \infty\right)\right), r(t)>0, r^{\prime}(t) \geq 0$;

(H3) $\tau \in C\left(\left[t_{0}, \infty\right), \mathbb{R}\right), \sigma \in C^{1}\left(\left[t_{0}, \infty\right), \mathbb{R}\right), \tau(t)<t, \sigma(t) \leq$ $\lambda t<t, \sigma^{\prime}(t)>0$, and $\lim _{t \rightarrow \infty} \tau(t)=\lim _{t \rightarrow \infty} \sigma(t)=$ $\infty$;

(H4) $f \in C(\mathbb{R}, \mathbb{R})$ and $f(x) / x \geq K>0$, for $x \neq 0$, and $K$ is a constant.

Further, we will consider the two cases

$$
\begin{aligned}
& \int^{\infty} \frac{1}{r(t)} d t=\infty, \\
& \int^{\infty} \frac{1}{r(t)} d t<\infty .
\end{aligned}
$$

By a solution of (1), we mean a real-valued function $x$ which satisfies (1) and $\sup \left\{|x(t)|: t \geq t_{x}\right\}>0$ for any $t_{x} \geq t_{0}$. Such a solution is said to be oscillatory if it has arbitrarily large zeros and nonoscillatory otherwise.

Neutral differential equations arise in a number of important applications in natural science and technology. For instance, they are used in problems dealing with vibrating masses attached to an elastic bar and in the study of distributed networks containing lossless transmission lines which appears in high speed computers where the lossless transmission lines are used to interconnect switching circuits; see Hale [1].

During the last 20 years, significant efforts have been devoted to investigate the oscillatory behaviour of neutral differential equations; see [1-16] and the references cited therein. In particular, (1) and related forms have been considered by several authors; see [10,11,13,14, 16]. Several recent results are surveyed in Sun et al. [13]. In addition, we refer to $[3-5,8]$, where the oscillatory behaviour of solutions of (1) with $n=2$ was studied.

In this paper, we establish oscillation theorems for solutions of (1). Our results generalize the results of Grammatikopoulos et al. [6] in some sense. Also, our results, in some sense, agree with the results of Sun et al. [13]. Here, we remove the restriction of [13] for $\tau$ and $\sigma$ to be commute. 


\section{Auxiliary Lemmas}

The following lemmas will be needed in the proof of our main results.

Lemma 1 (see [7, page 193]). Let $y(t)$ be an $n$ times differentiable function on $[0, \infty)$ of constant sign, let $y^{(n)}(t)$ be of constant sign and not identically equal to zero in any interval $\left[t_{0}, \infty\right), t_{0} \geq 0$, and let $y(t) y^{(n)}(t) \leq 0$. Then,

(i) there exists a $t_{1} \geq t_{0}$ such that $y^{(k)}(t), k=1, \ldots, n-1$, is of constant sign on $\left[t_{1}, \infty\right)$;

(ii) there exists an integer $l, 0 \leq l \leq n-1$, with $n-l$ odd, such that

$$
\begin{gathered}
y(t) y^{(k)}(t)>0, \quad k=0,1, \ldots, l, t \geq t_{1}, \\
(-1)^{n+k-1} y(t) y^{(k)}(t)>0, \quad k=l+1, \ldots, n-1, t \geq t_{1} .
\end{gathered}
$$

Lemma 2 (see [12]). Let $y(t)$ be a function as in Lemma 1. If

$$
y^{(n-1)}(t) y^{(n)}(t) \leq 0,
$$

then, for every $\theta \in(0,1)$, there exists a constant $M>0$, such that

$$
\left|y^{\prime}(\theta t)\right| \geq M t^{n-2}\left|y^{(n-1)}(t)\right|,
$$

for sufficiently large t.

Lemma 3 (see [2, page 169]). Let $y(t)$ be a function as in Lemma 1. If

$$
y^{(n-1)}(t) y^{(n)}(t) \leq 0,
$$

and $\lim _{t \rightarrow \infty} y(t) \neq 0$, then for every $\theta \in(0,1)$

$$
y(t) \geq \frac{\theta}{(n-1) !} t^{n-1} y^{(n-1)}(t),
$$

for sufficiently large t.

\section{The Main Result}

Theorem 4. Assume that (2) holds. If

$$
\int^{\infty} q(t) d t=\infty
$$

then every solution $x(t)$ of (1) is oscillatory.

Proof. Let $x(t)$ be a nonoscillatory solution of (1). Without loss of generality, we may assume that $x(t)$ is eventually positive (the proof is similar when $x(t)$ is eventually negative). That is, let $x(t)>0, x(\tau(t))>0$, and let $x(\sigma(t))>0$ for $t \geq t_{1} \geq t_{0}$.

Set

$$
z(t)=x(t)+p(t) x(\tau(t)) .
$$

Since $p(t)$ isnonnegative, $z(t)>x(t)>0$ for $t \geq t_{1}$.
From (1) and (11), we have

$$
\begin{aligned}
\left(r(t) z^{(n-1)}(t)\right)^{\prime} & =r(t) z^{(n)}(t)+r^{\prime}(t) z^{(n-1)}(t) \\
& =-q(t) f(x(\sigma(t)))<0 .
\end{aligned}
$$

Thus, $r(t) z^{(n-1)}(t)$ is decreasing and $z^{(n-1)}(t)$ is eventually of one sign. Hence, either

$$
z^{(n-1)}(t)>0, \quad \text { for } t \geq t_{2} \geq t_{1}
$$

or

$$
z^{(n-1)}(t)<0, \quad \text { for } t \geq t_{2} \geq t_{1} .
$$

If (14) holds, then

$$
r(t) z^{(n-1)}(t) \leq r\left(t_{2}\right) z^{(n-1)}\left(t_{2}\right)<0, \quad \text { for } t \geq t_{2} .
$$

Dividing this inequality by $r(t)$ and integrating from $t_{2}$ to $t$, then by using (2), we get

$$
\lim _{t \rightarrow \infty} z^{(n-2)}(t)=-\infty .
$$

This result along with (14) leads to $\lim _{t \rightarrow \infty} z(t)=-\infty$. But this contradicts the fact that $z(t)>0$. Thus, (13) holds. Then, from (12) and the fact that $r(t)$ is a positive nondecreasing function, we conclude that $z^{(n)}(t)<0$, for $t \geq t_{2}$. It follows that $z^{(i)}(t)(i=0,1, \ldots, n-1)$ is strictly monotonic and of constant sign eventually.

By applying Lemma 1, $z(t)$ satisfies (4) and (5). Since $n$ is even, the integer $l$ associated with $z(t)$ is odd; that is, $l \geq 1$. Hence, $z(t)$ is increasing for $t \geq t_{3} \geq t_{2}$.

Then, from (11) and the fact that $z(t)$ is increasing, we have

$$
\begin{gathered}
x(t)=z(t)-p(t) x(\tau(t)) \geq z(t)-p(t) z(\tau(t)) \\
\geq(1-p(t)) z(t) \geq\left(1-p_{0}\right) z(t), \\
\text { for } t \geq t_{3} .
\end{gathered}
$$

Let $t_{4} \geq t_{3}$ be such that $\sigma(t) \geq t_{3}$ for all $t \geq t_{4}$. Combining (H4) and (17), we get

$$
f(x(\sigma(t))) \geq K\left(1-p_{0}\right) z(\sigma(t)), \quad \text { for } t \geq t_{4} .
$$

It is clear that we can apply Lemma 2. Then, from (7) and the decreasing character of $z^{(n-1)}(t)$, we have

$$
\begin{gathered}
z^{\prime}(\sigma(t)) \geq \frac{M}{\lambda^{n-2}} \sigma^{n-2}(t) z^{(n-1)}\left(\frac{\sigma(t)}{\lambda}\right) \\
\geq M_{0} \sigma^{n-2}(t) z^{(n-1)}(t) \\
\text { for } t \geq t_{5} \geq t_{3},
\end{gathered}
$$

where $M_{0}=M / \lambda^{n-2}$.

Define

$$
w(t)=\frac{r(t) z^{(n-1)}(t)}{z(\sigma(t))},
$$

and then $w(t)>0$ for $t \geq t_{6}=\max \left\{t_{4}, t_{5}\right\}$. 
By differentiating $w$ and using (12), (18), and (19), we obtain

$$
\begin{aligned}
w^{\prime}(t) & =\frac{\left(r(t) z^{(n-1)}(t)\right)^{\prime}}{z(\sigma(t))}-\frac{\sigma^{\prime}(t) z^{\prime}(\sigma(t)) r(t) z^{(n-1)}(t)}{z^{2}(\sigma(t))} \\
& \leq-K\left(1-p_{0}\right) q(t)-\frac{M_{0} \sigma^{\prime}(t) \sigma^{n-2}(t)}{r(t)} w^{2}(t) .
\end{aligned}
$$

Since $r(t)>0$ and $\sigma^{\prime}(t)>0$, the term $\left(M_{0} \sigma^{\prime}(t) \sigma^{n-2}(t) /\right.$ $r(t)) w^{2}(t)>0$. Hence, $(21)$ reduces to

$$
w^{\prime}(t) \leq-K\left(1-p_{0}\right) q(t) .
$$

Integrating this inequality from $t_{7}$ to $t, t_{7}>t_{6}$, and using assumption (10), we see that $w(t) \rightarrow-\infty$ as $t \rightarrow \infty$. But this contradicts the positivity of $w(t)$. Hence, the theorem is proved.

In the above proof, being $l \geq 1$ plays an important role. In fact, $l=0$ is possible only for odd orders. In this case, the solutions are bounded. For unbounded solutions with $n$ being odd, the integer $l$ must be greater than or equal to 2 . Thus, it is easy to show that if $n$ is odd and the conditions of Theorem 4 are satisfied, then every unbounded solution of (1) is oscillatory.

Notice that if the solutions are assumed to be unbounded, then the restriction on $\sigma(t)$ in $(\mathrm{H} 2)$ can be improved to be $\sigma(t) \leq t$. Indeed, under the assumption of unboundedness, $z^{\prime}(t)$ is increasing. This modifies (19) as

$$
\begin{aligned}
z^{\prime}(\sigma(t)) & \geq z^{\prime}(\theta \sigma(t)) \geq M \sigma^{n-2}(t) z^{(n-1)}(\sigma(t)) \\
& \geq M \sigma^{n-2}(t) z^{(n-1)}(t), \quad \theta \in(0,1),
\end{aligned}
$$

where the rest of the proof stays as above.

Remark 5. The condition (10) can be rewritten as

$$
\int^{\infty} q(t)[1-p(\sigma(t))] d t=\infty .
$$

Here, there is no need for abounded value $p_{0}$ for the function $p(t)$; that is, $0 \leq p(t)<1$. When we take $n=2, r(t)=$ $1, f(x)=x$, and $\sigma(t)=t-\sigma_{0}$, we recover the results of Grammatikopoulos et al. [6]. In this case, we consider unbounded solutions.

Remark 6. Theorem 4 remains true if the function $f$ satisfies the condition that $x f(x)>0$ and there exists a nondecreasing function $\phi \in C\left(\left[t_{0}, \infty\right),(0, \infty)\right)$ with

$$
|f(x)| \geq \phi(|x|) .
$$

Theorem 7. Assume that (3) and (10) hold and $n \geq 4$ is even. Further, suppose that

$$
\int^{\infty}\left[C q(s)(\sigma(s))^{n-2} \delta(s)-\frac{1}{4 r(s) \delta(s)}\right] d s=\infty,
$$

where $C=\alpha K /(n-2) !, \alpha \in(0,1)$ is a constant, and $\delta(t)=\int_{t}^{\infty}(1 / r(s)) d s$. Then, every solution $x(t)$ of (1) either is oscillatory or tends to zero as $t \rightarrow \infty$.
Proof. Assume that (1) has a nonoscillatory solution $x(t)$. Without loss of generality, we assume that there exists a $t_{1} \geq$ $t_{0}$ such that $x(t)>0, x(\tau(t))>0$, and $x(\sigma(t))>0$ for all $t \geq t_{1}$.

Proceeding as in the proof of Theorem 4, we conclude that $r(t) z^{(n-1)}(t)$ is decreasing and $z^{(n-1)}(t)$ is eventually of one sign. Hence, either (13) or (14) holds.

If (13) holds, we obtain a contradiction by proceeding as in the proof of Theorem 4.

Suppose that (14) holds; that is, $z^{(n-1)}(t)<0$, for $t \geq$ $t_{2} \geq t_{1}$. Now, we consider two assumptions: unbounded solutions and bounded solutions.

If the solution $x(t)$ is unbounded, it is obvious that $z(t)$ is also unbounded. Since $z(t) z^{(n-1)}(t)<0$ and $n-1$ is odd, we have by Lemma 1 that $l \geq 2$ (if $l=0$, then $z(t)$ is bounded). Hence, from (4), we have that $z^{\prime}(t)>0$, and $z^{\prime \prime}(t)>0$. Therefore, $\lim _{t \rightarrow \infty} z(t)>0$.

Since $z(t)$ is increasing, we obtain

$$
x(t) \geq\left(1-p_{0}\right) z(t), \quad \text { for } t \geq t_{3} \geq t_{2} .
$$

By Lemma 3 and the fact that $z^{(n-2)}(t)$ is decreasing, we get

$$
z(\sigma(t)) \geq \frac{\theta[\sigma(t)]^{n-2}}{(n-2) !} z^{(n-2)}(t), \quad t \geq t_{4} \geq t_{2} .
$$

Combining (H4), (27), and (28), we obtain

$$
\begin{array}{r}
f(x(\sigma(t))) \geq C(\sigma(t))^{n-2} z^{(n-2)}(t), \\
\text { for } t \geq t_{5}=\max \left\{t_{3}, t_{4}\right\},
\end{array}
$$

where $C=\alpha K /(n-2)$ ! with $\alpha=\left(1-p_{0}\right) \theta \in(0,1)$.

Define

$$
w(t)=\frac{r(t) z^{(n-1)}(t)}{z^{(n-2)}(t)}
$$

and then $w(t)<0$ for $t \geq t_{5}$.

Differentiating $w$ and using (12) and (29), we obtain

$$
\begin{aligned}
w^{\prime}(t) & =\frac{\left(r(t) z^{(n-1)}(t)\right)^{\prime}}{z^{(n-2)}(t)}-\frac{w^{2}(t)}{r(t)} \\
& \leq-C q(t)(\sigma(t))^{n-2}-\frac{w^{2}(t)}{r(t)}
\end{aligned}
$$

Following $[8,13]$, we can show that $-1 \leq w(t) \delta(t)<0$. Indeed, since $r(t) z^{(n-1)}(t)$ is decreasing,

$$
r(s) z^{(n-1)}(s) \leq r(t) z^{(n-1)}(t), \quad s \geq t \geq t_{5} .
$$

Dividing by $r(s)$, then integrating from $t$ to $l$, and letting $l \rightarrow$ $\infty$, we get

$$
0 \leq z^{(n-2)}(t)+r(t) z^{(n-1)}(t) \delta(t) .
$$

Thus, we obtain

$$
-1 \leq \frac{r(t) z^{(n-1)}(t)}{z^{(n-2)}(t)} \delta(t)
$$


Hence,

$$
-1 \leq w(t) \delta(t)<0, \quad t \geq t_{5}
$$

Multiplying inequality (31) by $\delta(t)$ and integrating from $t_{5}$ to $t$, we get

$$
\begin{aligned}
& w(t) \delta(t)-w\left(t_{5}\right) \delta\left(t_{5}\right)+\int_{t_{5}}^{t} \frac{w(s)}{r(s)} d s+\int_{t_{5}}^{t} \frac{w^{2}(s) \delta(s)}{r(s)} d s \\
& \leq-\int_{t_{5}}^{t} C q(s)(\sigma(s))^{n-2} \delta(s) d s,
\end{aligned}
$$

or

$$
\begin{aligned}
w(t) & \delta(t)-w\left(t_{5}\right) \delta\left(t_{5}\right) \\
\leq & -\int_{t_{5}}^{t}\left[C q(s)(\sigma(s))^{n-2} \delta(s)-\frac{1}{4 r(s) \delta(s)}\right] d s \\
& -\int_{t_{5}}^{t} \frac{[w(s) \delta(s)+1 / 2]^{2}}{r(s) \delta(s)} d s .
\end{aligned}
$$

Thus,

$$
\begin{aligned}
w(t) \delta(t) \leq & -\int_{t_{5}}^{t}\left[C q(s)(\sigma(s))^{n-2} \delta(s)-\frac{1}{4 r(s) \delta(s)}\right] d s \\
& +w\left(t_{5}\right) \delta\left(t_{5}\right) .
\end{aligned}
$$

Using assumption (26), we see that $w(t) \delta(t) \rightarrow-\infty$ as $t \rightarrow$ $\infty$. But this contradicts (35).

If the solution $x(t)$ is bounded, then $z(t)$ is also bounded. Since $z(t) z^{(n-1)}(t)<0$ and $n-1$ is odd, we have by Lemma 1 that $l=0$ (otherwise, $z(t)$ is not bounded). Hence, from (4) and (5), we have

$$
(-1)^{i} z^{(i)}(t)>0, \quad i=0,1, \ldots, n-2 \text { for } t \geq t_{3} \geq t_{2} .
$$

From (11) and the fact that $z(t)>x(t)$, we obtain

$$
x(t) \geq z(t)-p_{0} x(\tau(t)) \geq z(t)-p_{0} z(\tau(t)),
$$

or

$$
x(t) \geq z(\tau(t))\left[\frac{z(t)}{z(\tau(t))}-p_{0}\right] .
$$

From (39) $z(t)>0, z^{\prime}(t)<0$, and $z^{\prime \prime}(t)>0$, we have $\lim _{t \rightarrow \infty} z(t)=\lambda \geq 0$. Now, we consider two cases.

Case I. Consider that $\lambda>0$. Since $z(t)$ is decreasing, there is an $\varepsilon>0$ and $t_{4} \geq t_{3}$ such that, for $t \geq t_{4}$,

$$
\lambda \leq z(t) \leq z(\tau(t)) \leq \lambda+\varepsilon .
$$

From this, we can conclude that

$$
\frac{z(t)}{z(\tau(t))} \geq \frac{\lambda}{\lambda+\varepsilon}
$$

Choose $t_{5} \geq t_{4}$ such that, for $t \geq t_{5}$, we have $p_{0}+\varepsilon_{1} \leq \lambda /(\lambda+\varepsilon)$, for some $\varepsilon_{1}>0$. Thus,

$$
\frac{z(t)}{z(\tau(t))} \geq p_{0}+\varepsilon_{1}, \quad t \geq t_{5} .
$$

Using this inequality in (41) and the fact that $z(t)$ is decreasing, we obtain

$$
x(t) \geq \varepsilon_{1} z(t), \quad t \geq t_{5} .
$$

By Lemma 3 and the fact that $z^{(n-2)}(t)$ is decreasing, we get

$$
z(\sigma(t)) \geq \frac{\theta[\sigma(t)]^{n-2}}{(n-2) !} z^{(n-2)}(t), \quad t \geq t_{6} \geq t_{3} .
$$

Combining (H4), (45), and (46), we obtain

$$
\begin{array}{r}
f(x(\sigma(t))) \geq C(\sigma(t))^{n-2} z^{(n-2)}(t), \\
\text { for } t \geq t_{7}=\max \left\{t_{5}, t_{6}\right\},
\end{array}
$$

where $C=\alpha K /(n-2)$ ! with $\alpha=\varepsilon_{1} \theta \in(0,1)$.

By using the transformation (30) and proceeding as in the previous assumption $(x(t)$ unbounded), again we obtain a contradiction with (26).

Case II. Consider that $\lambda=0$, since $x(t) \leq z(t), x(t)$ tends to zero as $t \rightarrow \infty$, and this completes the proof.

\section{Examples}

In this section, we present some examples to illustrate the above results.

Example 1. Consider the following even order nonlinear neutral differential equation:

$$
\begin{aligned}
& {\left[\sqrt{t}\left[x(t)+\left(\frac{1}{2}-\frac{1}{2 t+2}\right) x(t-3)\right]^{(n-1)}\right]^{\prime}} \\
& +t^{2} x\left(\frac{t-2}{2}\right)\left(1+\frac{1}{x^{2}((t-2) / 2)+1}\right)=0,
\end{aligned}
$$

$t>0$.

Here, $r(t)=\sqrt{t}, p(t)=(1 / 2-1 /(2 t+2)), q(t)=t^{2}, \tau(t)=t-3$, $\sigma(t)=(t-2) / 2$, and $f(x) / x \geq K=1$. We can see that all conditions of Theorem 4 are satisfied. Thus, every solution of (48) is oscillatory.

The function $p(t)=(1 / 2-1 /(2 t+2))$ in $(48)$ lies in the interval $(0,1 / 2)$; that is, $p_{0}=1 / 2<1$. Now, for the same equation, if $p(t)$ is replaced by $p(t)=(1-1 /(2 t+2))$, then there is no such $p_{0}<1$. In this case, by using condition (24), we conclude again that every solution of $(48)$ with $p(t)=(1-$ $1 /(2 t+2))$ is oscillatory.

Example 2. Consider the following nonlinear neutral differential equation:

$$
\left[e^{\alpha t}\left[x(t)+p_{0} x\left(t-\tau_{0}\right)\right]^{(n-1)}\right]^{\prime}+e^{\beta t} x(\lambda t)=0, \quad t>0,
$$

where $n \geq 4$ is even, $\tau_{0}>0,0<\lambda<1,0<p_{0}<1$, and $\beta \geq \alpha>0$. 
We can see that all conditions of Theorem 7 are satisfied. Thus, every solution of (49) either is oscillatory or tends to zero as $t \rightarrow \infty$.

Example 3. Consider the following even order nonlinear neutral differential equation:

$$
\begin{aligned}
{\left[e^{t}\left[x(t)+\frac{1}{a} x\left(\frac{t}{a}-n \ln a\right)\right]^{(n-1)}\right]^{\prime} } & \\
+\left(1-\frac{1}{a}\right) e^{(1+1 / b-1 / a) t} x\left(\frac{t}{b}\right) & =0 \\
t & >0,
\end{aligned}
$$

where $n \geq 4$ and $a>b>1$. It is easy to check that all conditions of Theorem 7 are satisfied. Thus, every solution of (50) either is oscillatory or tends to zero as $t \rightarrow \infty$. Indeed, $x(t)=e^{-t}$ is a solution that tends to zero as $t \rightarrow \infty$.

Note that the results of Sun et al. [13] cannot be applied in the above examples, since the $\tau$ and $\sigma$ are not commute.

\section{Conflict of Interests}

The authors declare that there is no conflict of interests regarding the publication of this paper.

\section{References}

[1] J. Hale, Theory of Functional Differential Equations, Springer, New York, NY, USA, 2nd edition, 1977.

[2] R. P. Agarwal, S. R. Grace, and D. O’Regan, Oscillation Theory for Difference and Functional Differential Equations, Kluwer Academic Publishers, Dordrecht, The Netherlands, 2000.

[3] J. Džurina, "Oscillation theorems for second order advanced neutral differential equations," Tatra Mountains Mathematical Publications, vol. 48, pp. 61-71, 2011.

[4] L. Erbe, T. S. Hassan, and A. Peterson, "Oscillation of second order neutral delay differential equations," Advances in Dynamical Systems and Applications, vol. 3, no. 1, pp. 53-71, 2008.

[5] S. R. Grace and B. S. Lalli, "Oscillation of nonlinear second order neutral differential equations," Rationalisation (Mathematics), vol. 3, pp. 77-84, 1987.

[6] M. K. Grammatikopoulos, G. Ladas, and A. Meimaridou, "Oscillations of second order neutral delay differential equations," Radovi Matematički, vol. 1, no. 2, pp. 267-274, 1985.

[7] G. S. Ladde, V. Lakshmikantham, and B. G. Zhang, Oscillation Theory of Differential Equations with Deviating Arguments, vol. 110, Marcel Dekker, New York, NY, USA, 1987.

[8] T. Li, B. Baculíková, and J. Džurina, "Oscillation results for second-order neutral differential equations of mixed type," Tatra Mountains Mathematical Publications, vol. 48, pp. 101-116, 2011.

[9] T. Li, Z. Han, P. Zhao, and S. Sun, "Oscillation of even-order neutral delay differential equations," Advances in Difference Equations, vol. 2010, Article ID 184180, 9 pages, 2010.

[10] T. Li and Y. V. Rogovchenko, "Asymptotic behavior of higherorder quasilinear neutral differential equations," Abstract and Applied Analysis, vol. 2014, Article ID 395368, 11 pages, 2014.
[11] F. Meng and R. Xu, "Oscillation criteria for certain even order quasi-linear neutral differential equations with deviating arguments," Applied Mathematics and Computation, vol. 190, no. 1, pp. 458-464, 2007.

[12] C. Philos, "A new criterion for the oscillatory and asymptotic behavior of delay differential equations," L'Académie Polonaise des Sciences. Bulletin, vol. 29, no. 7-8, pp. 367-370, 1981.

[13] Y. Sun, Z. Han, S. Sun, and C. Zhang, "Oscillation criteria for even order nonlinear neutral differential equations," Electronic Journal of Qualitative Theory of Differential Equations, vol. 30, pp. 1-12, 2012.

[14] G. Xing, T. Li, and C. Zhang, "Oscillation of higher-order quasilinear neutral differential equations," Advances in Difference Equations, vol. 2011, article 45, 2011.

[15] A. Zafer, "Oscillation criteria for even order neutral differential equations," Applied Mathematics Letters, vol. 11, no. 3, pp. 21-25, 1998.

[16] A. Zafer and R. S. Dahiya, "Oscillation of bounded solutions of neutral differential equations," Applied Mathematics Letters, vol. 6, no. 2, pp. 43-46, 1993. 


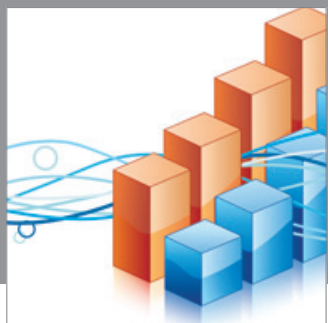

Advances in

Operations Research

mansans

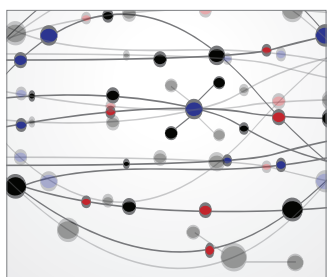

The Scientific World Journal
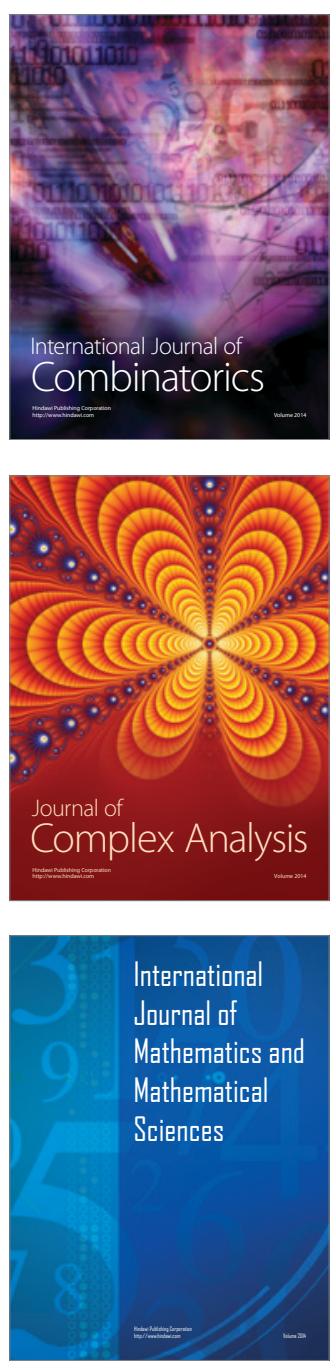
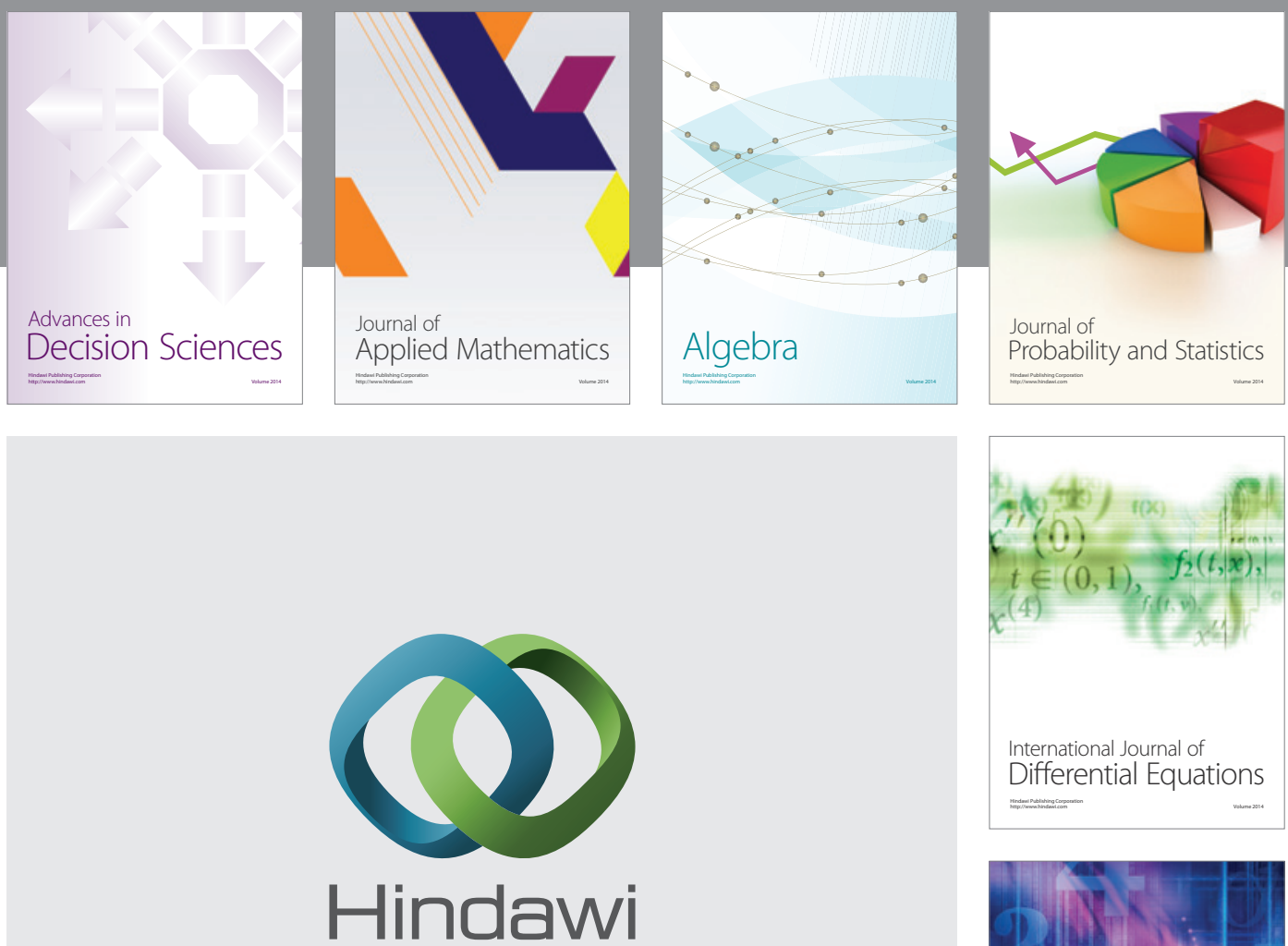

Submit your manuscripts at http://www.hindawi.com
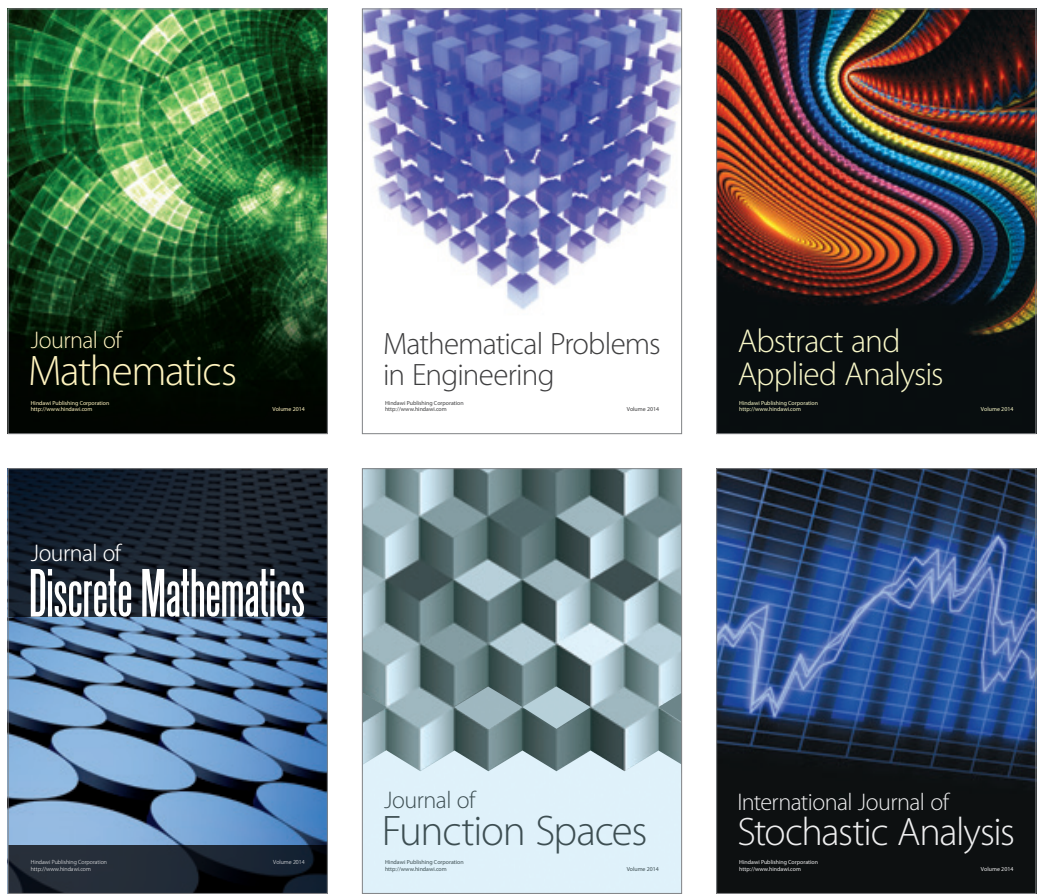

Journal of

Function Spaces

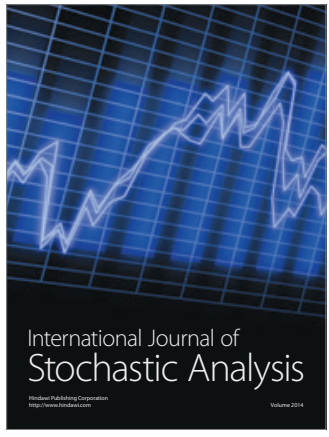

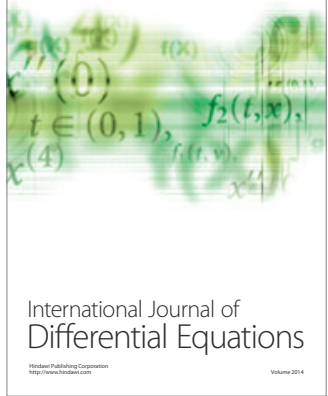
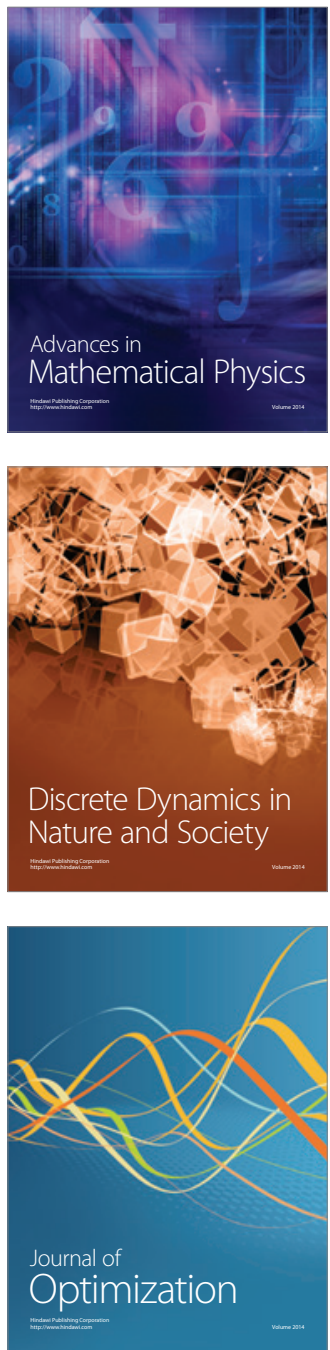